\title{
La doble transformación de un texto de Miguel de Cervantes en un poema alemán y en un Lied: el ensalmo de Preciosa "Cabecita, cabecita" de la novela ejemplar La gitanilla
}

\author{
The double Transformation of a text by Miguel de Cervantes in a German \\ Poem and in a Lied: The Precious Ensalmo "Cabecita, cabecita" of the \\ Nobel Exemplate La Gitanilla
}

\author{
Francisco Pérez Sánchez \\ Escuela Superior de Canto de Madrid \\ perezsanchezpianist@gmail.com
}

\begin{abstract}
RESUMEN
En este trabajo se va a exponer, describir y analizar la transformación de un texto cervantino extraído de la primera novela ejemplar de Cervantes, La gitanilla. En primer lugar, se estudiará la traducción al alemán realizada por Paul Heyse y publicada dentro de la colección de poesía castellana Spanisches Liederbuch (1852) de E. Geibel y P. Heyse. Y, en segundo lugar, se analizará la transformación en lied de la mano del compositor austriaco H. Wolf como el n ${ }^{\circ} 14$ de los Weltliche Lieder del Spanisches Liederbuch (1889-1890), bajo el título de Köpfchen, Köpfchen, nicht gewimmert. Se mostrarán las modificaciones y aportaciones del traductor al texto de la novela ejemplar, destacando los elementos originales — semánticos y formales_- que jugarán un papel crucial en la composición musical de Wolf. En la versión liederística se señalarán qué aspectos del poema español han permanecido y qué otros nuevos se han aportado, incidiendo en la traslación de las emociones contenidas en el texto poético mediante el diseño melódico, armónico, y la conformación de la parte vocal e instrumental.
\end{abstract}

Palabras clave: Lied, Cervantes, Hugo Wolf, Paul Heyse, Spanisches Liederbuch

\begin{abstract}
This article will present, describe and analyze the transformation of a text by Cervantes taken from his first exemplary novel, La gitanilla. First, we will study the German translation by Paul Heyse published in the Spanish poetry collection Spanisches Liederbuch (1852) by E. Geibel and P. Heyse. Second, we will analyze the transformation into lied by the Austrian composer H. Wolf as number 14 of the Weltliche Lieder of the Spanisches Liederbuch (1889-1890), the title of which is Köpfchen, Köpfchen, nicht gewimmert. We will present the changes and contributions that the translator made, highlighting the original elements - both semantic and formal - that play a crucial role in Wolf's musical composition. In the lied version, we will show which aspects of the Spanish poem have




\section{FranCisco PÉrez SÁNCHEZ}

remained and which new ones were contributed, focusing on the translation of the emotions contained in the poetic text through the melodic and harmonic concept, as well as the configuration of the vocal and instrumental part.

Key words: Lied, Cervantes, Hugo Wolf, Paul Heyse, Spanisches Liederbuch

Pérez Sánchez, F. (2017). La doble transformación de un texto de Miguel de Cervantes en un poema alemán y en un Lied: el ensalmo de Preciosa "Cabecita, cabecita" de la novela ejemplar $L a$ gitanilla. Cuadernos de Investigación Musical, 3, 5-19.

doi: http://dx.doi.org/10.18239/invesmusic.v0i3.1696

\section{INTRODUCCIÓN}

Desde finales del siglo XVIII se desarrolló en Alemania un creciente interés por la cultura y literatura españolas, como así lo demuestra la aparición de numerosos centros de estudios hispánicos en esa época (Weimar y Göttingen) y de investigadores sobre nuestra literatura (Johan Gottfried Herder, Johann Andreas Dieze, Jakob Grimm, los hermanos Schlegel y Ludwig Tieck). Gracias a su labor, comenzaron a proliferar en tierras germánicas las traducciones de las obras más importantes de los escritores del siglo de oro. De entre todas ellas, el Quijote de Cervantes se erigió como la principal obra traducida en el romanticismo alemán. Igualmente, las Novelas Ejemplares disfrutaron de una gran acogida, siendo traducidas en numerosas ocasiones a lo largo del siglo XIX. Una de las más conocidas fue La gitanilla, popularizada en ese país gracias a la pieza de teatro Preciosa (1821) de Pius Alexander Wolff con música de Carl Maria von Weber.

Al final de este periodo se publicó el Spanisches Liederbuch (1852) o Cancionero Español de Emanuel Geibel (1815-1884) y Paul Heyse (1830-1915) en Berlín, el cual alberga una selección de poemas anónimos del siglo XIV al XVII, y de los más grandes escritores del siglo de oro español (San Juan de la Cruz, Lope de Vega, Cervantes, etc.) traducidos al alemán. Estos traductores recopilaron los textos procedentes de diferentes fuentes y colecciones; la principal, la Floresta de Rimas Antiguas Castellanas de Johann Nikolaus Böhl von Farber, publicada por Perthes y Besser en Hamburgo en tres partes entre 1821 y 1825 (Bohl de Barber, 1821-1825). El Spanisches Liederbuch disfrutó de gran éxito y difusión, sobre todo entre los compositores de canción o lied como Schumann, Cornelius, Brahms, Jensen, etc. y, en especial, por parte del autor austriaco que hoy nos ocupa, Hugo Wolf (18601903), que compuso 44 lieder sobre poemas de esta colección, recogiéndolos bajo el mismo título: Spanisches Liederbuch (1889-1890).

El único texto traducido de Cervantes de la colección de Geibel y Heyse fue el utilizado por $\mathrm{H}$. Wolf para componer uno de los ejemplos más peculiares de la recepción musical de la obra cervantina en un país de habla germánica. La versión de Paul Heyse de 
LA DOBLE TRANSFORMACIÓN DE UN TEXTO DE MIGUEL DE CERVANTES EN UN POEMA ALEMÁN Y EN UN LiED: El ENSALMO DE PRECIOSA “CABECITA, CABECITA" DE LA NOVELA EJEMPlaR La GITANILLA

este poema, extraído de la primera novela ejemplar de Cervantes La gitanilla ${ }^{1}$, se transforma en lied de la mano del compositor austriaco como el no 14 de los Weltliche Lieder [Canciones profanas] bajo el título de Köpfchen, Köpfchen, nicht gewimmert [Cabecita, cabecita, no gimotees] (Wolf, 1967/1994: 94-95).

En este estudio se va a exponer, describir y analizar la transformación de este breve texto cervantino en un poema alemán, primero, y en un lied, después. Se mostrarán las modificaciones y aportaciones del traductor al texto de la novela ejemplar, destacando los elementos originales —semánticos y formales-, que jugarán un papel crucial en la composición musical de Wolf. En la versión liederística se señalarán qué aspectos del poema español han permanecido y qué otros nuevos se han aportado, incidiendo en la traslación de las emociones contenidas en el texto poético mediante el diseño melódico, armónico y la conformación de la parte vocal e instrumental ${ }^{2}$.

\section{Primera transformación}

Este poema no es recitado, como lo son otros que aparecen en la novela ejemplar, sino que Preciosa lo escribe en un papel por requerimiento del padre de su admirador, Don Juan de Cárcamo, que pretende convertirse en gitano para conseguir su amor. Le ha susurrado unas palabras al oído para calmarlo del vahído causado por los celos al ser leído un soneto dedicado a ella, y como no puede repetirlas, porque desvelaría el plan de su adorador, ingenia este breve conjuro para contentar al padre ${ }^{3}$. En palabras de Preciosa, este ensalmo sirve "para preservar el mal del corazón y los vaguidos de cabeza" (Cervantes Saavedra, 1980/2010: 97).

Con el objetivo de comprender adecuadamente este proceso o cadena de traducción, se hace necesario analizar brevemente el poema cervantino. El texto constituye una copla mixta de pie quebrado (Montero Reguera, 2013: 36) formada por versos que contienen ocho o cuatro sílabas. El poema contiene una sola estrofa con trece versos. Los cuatro primeros, de ocho sílabas cada uno, forman una redondilla, es decir con rima consonante de tipo "abba" y, a continuación, le sigue una especie de "tercetillo" monorrimo de versos con cuatro sílabas (aaa). Las siguientes seis líneas riman en parejas, con un verso corto de cuatro (de pie quebrado) y otro de ocho (ccddee). Un poema de carácter popular, en el que Cervantes imita magistralmente el estilo de coplas y romances para recrear una especie de oración aparentemente ingenua y algo infantil. A ello contribuye la utilización del diminutivo y de un léxico sencillo, sumado a la aliteración del segundo verso ("tente en ti, no te resbales"). En cuanto al ritmo, el poema español, siempre fluido y ligero, principia la

\footnotetext{
${ }^{1}$ Probablemente, el traductor, Paul Heyse, tomó el texto del poema de la antología recopilada por Huber que circulaba en esa época en Alemania (HUBER, 1832).

2 Existen otros dos ejemplos de lieder alemanes compuestos utilizando la traducción de Heyse del texto cervantino. La primera es de Peter Cornelius (1824-1874) de 1854-55, y, la segunda, de Julius Hermann Krigar (1819-1880) de 1866.

${ }^{3}$ Cervantes Saavedra, 1613; Cervantes Saavedra,1799: 1-182; Cervantes Saavedra, 2003: 623.
} 


\section{FrANCISCo PÉREZ SÁNCHEZ}

mayoría de sus versos con un anapesto, los cuales albergan, en general, dos pies métricos en los de ocho sílabas, y uno en los de cuatro (ejemplo 1).

\begin{tabular}{ll} 
Cabecita, cabecita & Köpfchen, Köpfchen, nicht gewimmert, \\
tente en ti, no te resbales, & Halt' dich wacker, halt' dich munter, \\
y apareja dos puntales & Stütz' zwei gute Säulchen unter, \\
de la paciencia bendita. & Heilsam aus Geduld gezimmert! \\
Solicita & Hoffnung schimmert, \\
la bonita & Wie sich's aus verschlimmert \\
confiancita; & Und dich kümmert. \\
no te inclines & Mußt mit Grämen \\
a pensamientos ruines; & Dir nichts zu Herzen nehmen, \\
verás cosas & Ja kein Märchen, \\
que toquen en milagrosas, & Daß zu Berg dir stehn die Härchen; \\
Dios delante & Da sei Gott davor \\
y San Cristóbal gigante. & Und der Riese Chistophor! \\
\multicolumn{1}{c}{ Ejemplo 1: Poema original y traducción de Heyse. }
\end{tabular}

El breve ensalmo de Preciosa aparece como el n ${ }^{\circ} 90$ (de un total de 99 poemas profanos traducidos) bajo el título de Köpfchen, Köpfchen, nicht gewimmert (Preciosa's Sprüchlein gegen Kopfweh) [Cabecita, cabecita, no gimotees. Discursito de Preciosa contra el dolor de cabeza] (Spanisches Liederbuch, Weltliche Lieder $\mathrm{n}^{\circ}$ 90), traducido por Paul Heyse (Geibel y Heyse, 1852: 162).

$\mathrm{Al}$ aislar el poema del contexto de la novela se pierde la broma o la ingeniosidad de su alumbramiento, que ya he aclarado previamente. La traducción solamente pretende conservar el carácter sencillo y popular del ensalmo u oración. En cuanto a la métrica, Heyse no logra cuadrar perfectamente la longitud de los versos del poema español, formada por versos que contienen ocho o cuatro sílabas. En la traducción, los versos $n^{\circ} 6$, 9 y 12 no corresponden exactamente al número de sílabas del español, como podemos comprobar en la siguiente tabla, lo cual provoca la pérdida de la perfección y equilibrio formal del texto.

\begin{tabular}{|l|l|}
\hline $\mathbf{N}^{\circ}$ sílabas del poema original & 8888444484848 \\
\hline $\mathbf{N}^{\circ}$ sílabas de la traducción de Heyse & 8888464474868 \\
\hline
\end{tabular}

Tabla 1: Longitud de los versos del poema original y de la versión de Heyse. 
LA DOBLE TRANSFORMACIÓN DE UN TEXTO DE MIGUEL DE CERVANTES EN UN POEMA ALEMÁN Y EN UN LiED: EL ENSALMO DE PRECIOSA “CABECITA, CABECITA” DE LA NOVELA EJEMPlaR La GITANILLA

La rima entre versos se mantiene análoga, pero se pierden los diminutivos de nuestro idioma que contribuyen al carácter íntimo, cariñoso e ingenuo del texto de Cervantes. Los acentos rítmicos entre ambas versiones son totalmente diferentes, pues la traducción de Heyse obedece a una regularidad más trocaica. Destaca la coincidencia rítmica en el inicio de la línea no 2: "Hallt' dich wacker, halt' dich munter" por "tente en tị, no te resbales"; y la igualdad en el verso $\mathrm{n}^{\circ} 7$ si no se acentúa la conjunción "und": "Und dich kümmert" por "confiancita". El resto de los versos difieren en cuanto al ritmo.

Como podemos comprobar, el texto cervantino presenta un reto para Heyse, que finalmente decide resolver ideando un poema casi totalmente nuevo, inspirado en el del autor alcalaíno. El traductor alemán toma los ingredientes semánticos del original para intentar conservar la estructura formal y rima, ya que el ritmo se le presenta tarea imposible, utilizándolos y alterando su sentido exacto con la intención de producir una creación lírica que suponga un paralelo alemán a lo que el español representa. Al menos, el principio y final de la traducción constituyen dos puntos en los que se recrea el léxico del español de manera más fiel: "Köpfchen, Köpfchen" por "Cabecita, cabecita” y "Da sei Gott davor/ und der Riese Christofor" por "Dios delante/ y San Cristóbal gigante", que significan exactamente lo mismo. Heyse incorpora elementos semánticos que el poema español no contiene como "nicht gewimmert" [no gimotees] o "Halt' dich wacker, halt' dich munter" [se valiente, se alegre], y sustituye el sustantivo "confiancita" por el de "Hoffnung" [esperanza]. Según Susan Youens, este último cambio es un ejemplo característico del deseo de los traductores del Cancionero Español de engranar una paráfrasis donde la traducción del modismo español parece imposible 4 . También resulta curiosa la traducción de "verás cosas/ que toquen en milagrosas" por "Ja kein Märchen, / daß qu Berg dir stehen die Härchen" [no es un cuento/que te ponga los pelos de punta], transformándose el sentimiento de impresión ante algo sorprendente por el de miedo ante una historia o cuento (ejemplo 2). Como bien afirma Youens: "In Heyse's translation, the naiveté becomes Teutonic, miracles turned into Märchen and hair standing on end to the tune of a German idiom" (Youens, 1992: 236).

\footnotetext{
4 'The sleight of hand by which the idiomatic expression 'Solicita/la bonita/confiancita'- 'confiancita' a very personal and intimate confidence- becomes the Germanic invocation of hope is characteristic of Heyse's and Geibel's willingness to engage in paraphrase where an idiom was, they felt, untranslatable" (Youens, 1992: 237).
} 


\section{FrANCISCo PÉREZ SÁNCHEZ}

$\begin{array}{ll}\begin{array}{l}\text { Cabecita, cabecita } \\ \text { tente en ti, no te resbales, }\end{array} & \text { iCabecita, cabecita, no gimotees, } \\ \text { y apareja dos puntales } & \text { apóyate en dos buenas columnitas, } \\ \text { de la paciencia bendita. } & \text { curativas de paciencia construidas! } \\ \text { Solicita } & \text { La esperanza brilla, } \\ \text { la bonita } & \text { Cuando fuera se empeora } \\ \text { confiancita; } & \text { y te aflige. } \\ \text { no te inclines } & \text { Las penas no debes } \\ \text { a pensamientos ruines; } & \text { tomártelas a pecho, } \\ \text { verás cosas } & \text { no es un cuento } \\ \text { que toquen en milagrosas, } & \text { que te ponga los pelos de punta; } \\ \text { Dios delante } & \text { ¡Dios delante } \\ \text { y San Cristóbal gigante. } & \text { y el gigante Cristóbal! }\end{array}$

Ejemplo 2: Poema original y traducción de la versión de Heyse

En conclusión, en esta primera transformación, el breve y encantador ensalmo cervantino se convierte en un poema alemán que se desprende de su carácter popular para adquirir un cierto aire de cuento y fantasía más propio del romanticismo alemán, eso sí, intentando conservar la estructura formal del original.

\section{SEGUNDA TRANSFORMACIÓN}

No hay constancia del conocimiento por parte de Wolf de la novela ejemplar de Cervantes, La gitanilla, en la que se encuentra contenido este poema, pero en cambio sí se sabe de su admiración por escritor alcalaíno y de su Quijote. En esta segunda transformación del poema de Preciosa, tanto la estructura del poema como su contenido se convierten en la principal obsesión de Wolf a la hora de componer el lied ${ }^{6}$. En el análisis musical de los parámetros generales de la canción, observamos como todos colaboran en la plasmación del contenido poético. La tonalidad, sib mayor, en Wolf puede transmitir la

\footnotetext{
${ }^{5}$ En la biblioteca privada de Hugo Wolf se encontraban, entre otros, el Quijote de Cervantes y varios volúmenes de obras dramáticas de autores españoles como Tirso de Molina, Calderón, Lope de Vega y el propio Cervantes (Bachleitner, 2010: 52).

${ }^{6}$ Köpfchen, Köpfchen, nicht gewimmert (Preciosa's Sprïchlein gegen Kopfweh). Spanisches Liederbuch, $\mathrm{n}^{\circ} 24$ (Weltliche Lieder $\mathrm{n}^{\mathrm{o}}$ 14). Fecha y lugar de composición: 31 de octubre de 1889, Perchtoldsdorf. Primera noticia de su ejecución e intérpretes: 22 de octubre de 1890 en Mainz, ejecución privada del compositor para Ludwig Strecker y su mujer (Jestremski, 2011: 386, 395).
} 


\section{La DOBLE TRANSFormación DE UN TEXTO DE Miguel De CERVANTES EN UN POEMA ALEMÁN Y EN UN LIED: El ENSALMo DE PRECIOSA “CABECITA, CABECITA" DE LA NOVEla EJEMPLAR La GITANILLA}

ingenuidad y sencillez que caracterizan el estilo del texto, pero, igualmente, la circunstancia de un amor nuevo, que también está presente en el argumento de la novela ${ }^{7}$.

El compás y ritmo, 4/8, que establece a una declamación basada en el valor de corchea en la parte vocal y un ritmo repetitivo en la parte pianística, cuadra perfectamente con el ritmo trocaico del texto. La indicación de tempo, Mäßig [moderato], marca el fluir tranquilo de las corcheas. En cuanto a la dinámica y articulación, Wolf apunta una precisa matización que abarca intensidades dinámicas desde el $p p$ hasta el $f$, junto a numerosos reguladores. Paralelamente, la composición contiene un variado catálogo de articulaciones musicales que apoyan o complementan el sentido del poema ${ }^{8}$.

La estructura formal corresponde perfectamente a la división interna del poema y a su contenido. El lied se singulariza por una fuerte unidad y coherencia debidas a su regularidad rítmica y temática. La estructura seguiría el siguiente patrón: A B A’ C.

En la siguiente tabla se puede apreciar claramente la relación entre la estructura del poema, las secciones del lied y las tonalidades que atraviesa (tabla 2).

\begin{tabular}{|c|c|c|c|}
\hline Poema & Sección & Compases & Tonalidad \\
\hline $\begin{array}{l}\text { Köpfchen, Köpfchen, nicht gewimmert, } \\
\text { Halt' dich wacker, halt' dich munter, } \\
\text { Stütr' ₹wei gute Säulchen unter, } \\
\text { Heilsam aus Geduld gezimmert! }\end{array}$ & $\begin{array}{c}\text { Preludio } \\
\text { A }\end{array}$ & $\begin{array}{c}1-2 \\
3-11\end{array}$ & $\begin{array}{c}\text { Sib m } \\
\text { Sib m-Sib M } \\
\text { Sol M }\end{array}$ \\
\hline $\begin{array}{l}\text { Hoffnung schimmert, } \\
\text { Wie sich's aus verschlimmert } \\
\text { Und dich kümmert. } \\
\text { Mußt mit Grämen } \\
\text { Dir nichts zu Herzen nebmen, }\end{array}$ & $\begin{array}{c}\text { Enlace } \\
\text { B }\end{array}$ & $\begin{array}{c}12 \\
13-18\end{array}$ & Sol M \\
\hline $\begin{array}{l}\text { Ja kein Märchen, } \\
\text { Daß zu Berg dir stehn die Härchen; }\end{array}$ & $A^{\prime}$ & $23-26$ & Sib m \\
\hline $\begin{array}{l}\text { Da sei Gott davor } \\
\text { Und der Riese Christophor! }\end{array}$ & $\mathrm{C}$ & $27-34$ & Sib M \\
\hline & Postludio & $35-40$ & Sib M \\
\hline
\end{tabular}

Tabla 2: Correspondencia formal y tonal entre el poema y el lied

\footnotetext{
${ }^{7}$ La tesis doctoral de Jarosch aborda el estudio del significado de cada tonalidad en la obra de Hugo Wolf (JAROSCH, 1927: 141-151).

${ }^{8} \mathrm{Al}$ final de este artículo adjuntamos la partitura completa del lied, lo que permitirá observar perfectamente todos los elementos analizados.
} 


\section{FrANCISCO PÉREZ SÁNCHEZ}

Tanto el diseño armónico y melódico como la construcción de la parte vocal y pianística tienen como fin destacar, mostrar y expresar las emociones y sentimientos del texto traducido. Desde el punto de vista armónico, en esta composición Wolf recurre a diferentes herramientas típicas de su estilo, combinando cromatismo y diatonismo en aras de una coherente expresividad que refleje el sentido poético. Para tal fin aplica diferentes recursos armónicos como la ambigüedad tonal, la modulación a la mediante, sextas aumentadas, séptimas disminuidas, acordes mixtos, elementos estáticos, etc.

La citada inestabilidad tonal se establece desde el primer compás, en el que el quinto grado ejecuta un ostinato en ritmo de corchea a modo de pedal, formando con el bajo (sexto grado rebajado o novena de la dominante) una séptima mayor y una segunda menor en la parte débil del compás, que produce una sonoridad chocante o de cluster. El efecto taladrante de este intervalo, junto a su tesitura aguda, simboliza musicalmente los pinchazos de un dolor de cabeza. En la línea vocal se adivina más claramente la estructura armónica de esta primera frase, que en el compás quinto se desarrolla bajo la escala de sib menor y en el compás sexto, en las palabras "balt' dich munter" [sé alegre], dibuja el acorde de tónica de sib mayor. Con esta encantadora candidez armónica, Wolf cumple con el paralelismo sintáctico de estos versos, "Halt' dich wacker, halt'dich munter" [sé valiente, sé alegre], yuxtaponiendo los modos mayor y menor. Tras este inicio desestabilizador, en el compás 9 se elabora una cadencia en sol mayor con su acorde de séptima de sensible, que nos mantiene en una relajante armonía diatónica hasta el final de la sección A coincidiendo con las palabras "heilsam aus Geduld gerimmert!" [curativas de paciencia construidas].

En la sección $\mathrm{B}$ nos sigue sorprendiendo el ingenio de nuestro compositor, que descubre nuevos procedimientos armónicos para aplicarlos en la estructuración tonal del lied. Donde en el poema encontramos el "tercetillo" monorrimo que trata de la esperanza ante situaciones dificultosas y preocupantes, Wolf crea una estructura armónica cíclica o en bucle que repite tres veces seguidas, provocando una sensación de círculo cerrado sin salida, empleando acordes disminuidos y una armonía altamente cromática. En la segunda parte de esta sección $\mathrm{B}$, el círculo vicioso se rompe por medio de una repentina modulación a do\# menor. Este momento, en el que la dinámica del lied desciende a un $\not p$, coincide con las palabras "Las penas no debes/tomártelas a pecho". El contraste con una tonalidad menor, que se encuentra a distancia de tritono de sol mayor, crea un efecto de color sonoro de oscuridad que nos conecta con el significado del sustantivo "penas".

En la sección de 4 compases que hemos denominado A' (cc. 23-26), se reproduce el estado de ambigüedad tonal. A partir del compás 27, parte $C$, la armonía se vuelve más diatónica, aunque Wolf sigue introduciendo elementos de color con algunos acordes mixtos y cromáticos, como en la palabra "Riese" [gigante]. En la última sílaba del nombre de San Cristóbal (Christophor) se crea el efecto de una suspensión agógica mediante una fermata y una semicadencia en la séptima de dominante del cuarto grado (mib mayor). El final de la sección $\mathrm{C}$ continúa con una reafirmación de la tonalidad principal (sib mayor) al igual que en el postludio pianístico. 
LA DOBLE TRANSFORMACIÓN DE UN TEXTO DE MIGUEL DE CERVANTES EN UN POEMA ALEMÁN Y EN UN LiED: El ENSALMO DE PRECIOSA “CABECITA, CABECITA" DE LA NOVELA EJEMPlaR La GITANILLA

Esta tranquilidad tonal parece simbolizar el final del tormento, como si el conjuro hubiese surtido el efecto deseado. Este retraso de la tónica hasta el final del lied constituye uno de los elementos que mejor demuestran la correspondencia entre la estructura musical y el texto.

En cuanto a la parte vocal, su tesitura se sitúa entre las notas fa4 y sol5. Aunque aparentemente cómoda vocalmente, el ámbito en el que se mueve, constantemente alrededor de la zona de paso vocal (fa5), hacen de esta canción una de las más incómodas desde el punto de vista de la técnica del canto. El valor declamatorio básico por sílabas del texto se fundamenta en la corchea. Únicamente se utiliza la semicorchea en nota de paso sobre una misma sílaba y el valor de negra en las síncopas, con el fin de exagerar la declamación. El carácter de conjuro o plegaria casa adecuadamente con la reiteración de sonidos, que transcriben la naturaleza insistente del deseo de que desaparezca el dolor de cabeza. A este propósito colabora la construcción más diatónica de la parte pianística, que le otorga el papel mitigador de este suplicio. No se ha prescrito silencio alguno en la frase vocal que va de los compases 3 al 11 porque, seguramente, en lo que también coincide Youens, la protagonista "adapts her words to the rhythmic pounding of her headache and perhaps as well because the words/vocal line are her weapon against the pain" (Youens, 1992: 239).

Melódicamente, la sección B se distingue de la anterior por el empleo de síncopas como en la palabra "Hoffnung" [esperanza] - por la menor repetición de notas y por la finalización de los versos por salto de quinta descendente. En el décimo verso o sección A' regresa la reiteración de sonidos, pero esta vez se sustituyen los intervalos de segunda menor por saltos de séptima y novena. La declamación se exagera de esta manera intentando ser coherente con el contenido del texto, en el que se refiere al efecto producido por los cuentos fantásticos que ponen los pelos de punta. En los dos últimos versos, que Wolf repite para enfatizar el final del lied, el canto obedece a los mismos principios utilizados en toda la composición (repetición de notas y descenso cromático) pero a la manera de un himno o alabanza. Además, aparece uno de los pocos melismas a los que Wolf recurre en el Spanisches Liederbuch: en la palabra "Christophor" (en la primera aparición de dicho nombre Wolf lo subraya mediante una fermata).

Una de las peculiaridades llamativas de la escritura pianística de esta composición reside en su tesitura: todo el lied se encuentra escrito en la parte aguda del piano, es decir, desde el do central hacia la derecha del teclado. Este procedimiento es utilizado por Wolf para caracterizar la música de cierto aire fantástico o juguetón, como en Elfenlied o en Mausfallen-Sprüchlein (Mörike-Gedichte) ${ }^{10}$. La mano derecha repite impertérrita una nota en

\footnotetext{
${ }^{9}$ Este recurso melódico aparece raramente en la obra vocal de Wolf y, cuando lo hace, siempre persigue un fin artístico que enfatice el texto (Thürmer, 1970:129).

${ }^{10}$ Youens también opina que este aspecto de su composición recuerda a los lieder anteriores sobre texto de Mörike: "a lied reminiscent of the earlier Mörike song 'Mausfallen-Sprüchlein' in its sophisticated play on naiveté” (Youens, 1992: 239).
} 


\section{FrANCISCo PÉREZ SÁNCHEZ}

valor de corchea que, a partir del compás 9, se convierte en octava y, a partir del 27, en acordes; en el postludio regresa a la octava y, finalmente, a una sola nota como al inicio.

En la primera parte de la sección A, que ya hemos descrito como altamente cromática y disonante, la mano izquierda ejecuta saltos en staccato formando una segunda menor con la mano derecha en la parte débil del compás ${ }^{11}$. Esta escritura a dos voces, de textura clara pero ácida debido al choque de semitono entre las dos manos junto al insistente ritmo de corchea, describe musicalmente la sensación que producen cierto tipo de dolores de cabeza, a modo de pinchazos ${ }^{12}$. La parte que acompaña a los dos últimos versos (cc. 27-34) de textura homofónica a modo de música de órgano, intenta enfatizar las palabras del texto que nombran a Dios y al santo Cristóbal. El postludio pianístico transmite un progresivo apaciguamiento de la tensión y la solemnidad del final, disminuyéndose la intensidad del $p$ al $p p$. La mano izquierda interpreta un diseño en dos partes totalmente diatónico y conclusivo que consiste en una melodía en notas dobles, como una sonoridad de trompa, que simboliza el final del tormento del dolor.

\section{ConClusiones}

Pese a que Köpfchen, Köpfchen, nicht gewimmert podría haber estado entre los poemas que la editorial Schott consideró "unsuitable for music", Wolf aprovecha su simplicidad para convertirlo en un ejemplo de ingenuidad, sencillez y broma musical. La fidelidad al texto constituye, de nuevo, el paradigma del estilo del maestro vienés: la caracterización del dolor de cabeza en el piano con el choque de la dominante con su segunda menor, el progresivo efecto del ensalmo, que poco a poco convierte la ambigüedad tonal en una afirmación de la tónica hacia el final de la composición, y la invocación a Dios y a San Cristóbal en una escritura y canto más homofónicos a modo de himno o invocación, son ejemplos de este perfecto maridaje entre palabra y música. Werba considera los medios compositivos empleados por Wolf en este lied más bien económicos ${ }^{13}$.

Resulta sorprendente que un aspecto que no aparece en la novela de Cervantes, el dolor de cabeza, se convierta, por invención del traductor Heyse, en la seña de identidad de esta canción. En el presente caso, esta cadena en la traducción en la que se pierden y ganan peculiaridades semánticas, el invento de una escena en la que una joven recita un conjuro permite a Wolf idear una magistral composición de efectos sensoriales en la caracterización del dolor y su antídoto, mediante una oración.

\footnotetext{
${ }^{11}$ Este ritmo regular en staccato en parte débil junto al intervalo de semitono se encuentra asociado, según la clasificación de Sams, a la idea de infantilismo, fragilidad y pequeñez (motivos 2 y 3) (Sams, 1983: 18-19). 12 A este respecto, Sams opina de la misma manera: "The accompaniment's clashing semitones in single quavers suggest a series of tiny but persistent pangs" (Sams, 1983: 279).

13 "Sparsamer in der Wahl der Mittel, transparent in der Deutung von Kopfschmerzen" [Económico en la elección de los medios, transparente en la interpretación del dolor de cabeza] (Werba, 1984: 157).
} 
La DOBLE TRANSFORMACIÓN DE UN TEXTO DE Miguel De CERVANTES EN UN POEMA ALEMÁN Y EN UN LiED: El ENSALMO DE PRECIOSA “CABECITA, CABECITA" DE LA NOVELA EJEMPlaR La GITANILLA

A

singstimme. $\frac{\text { Mässig. }}{Q^{2}}$ Si $b \mathrm{~m}$

Klavier.
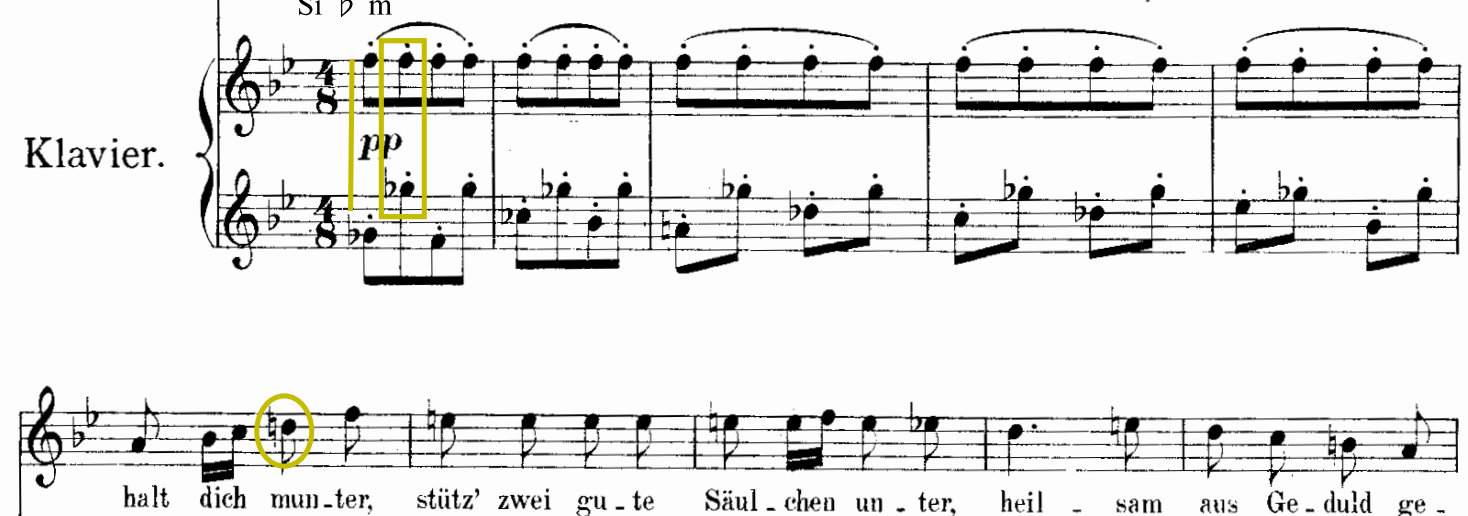

Si b M

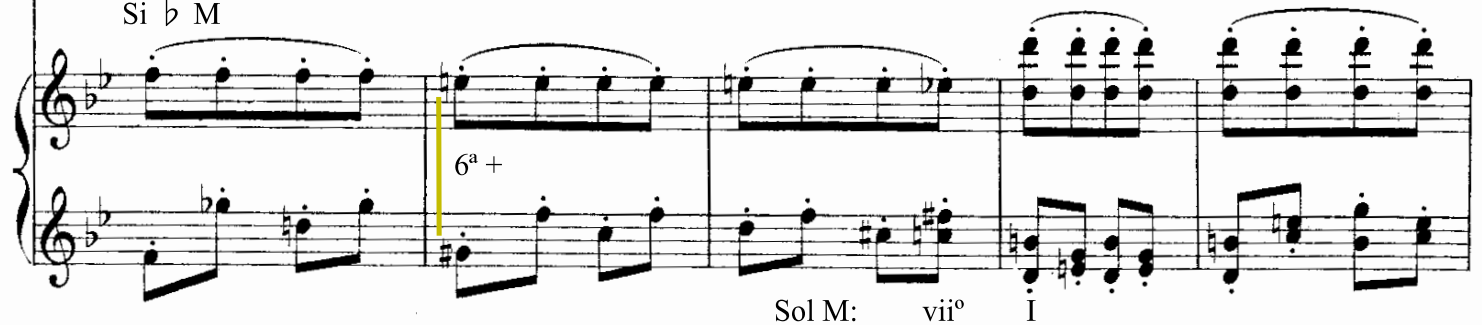

B
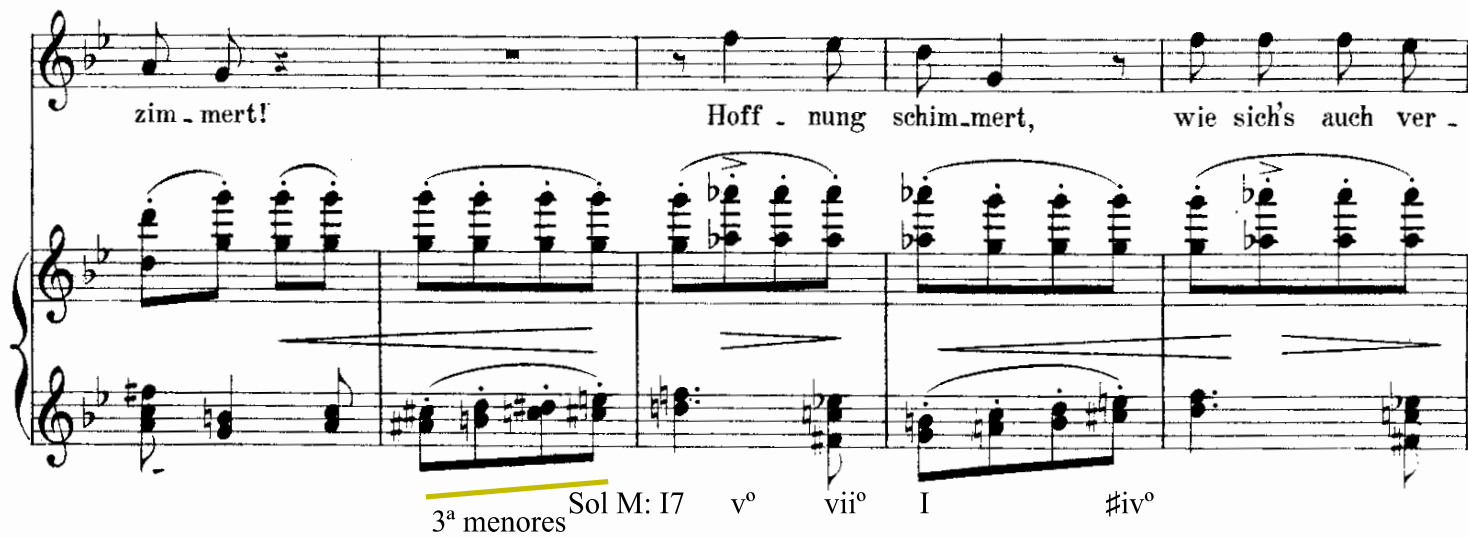
Francisco PÉREz SÁNCHEZ

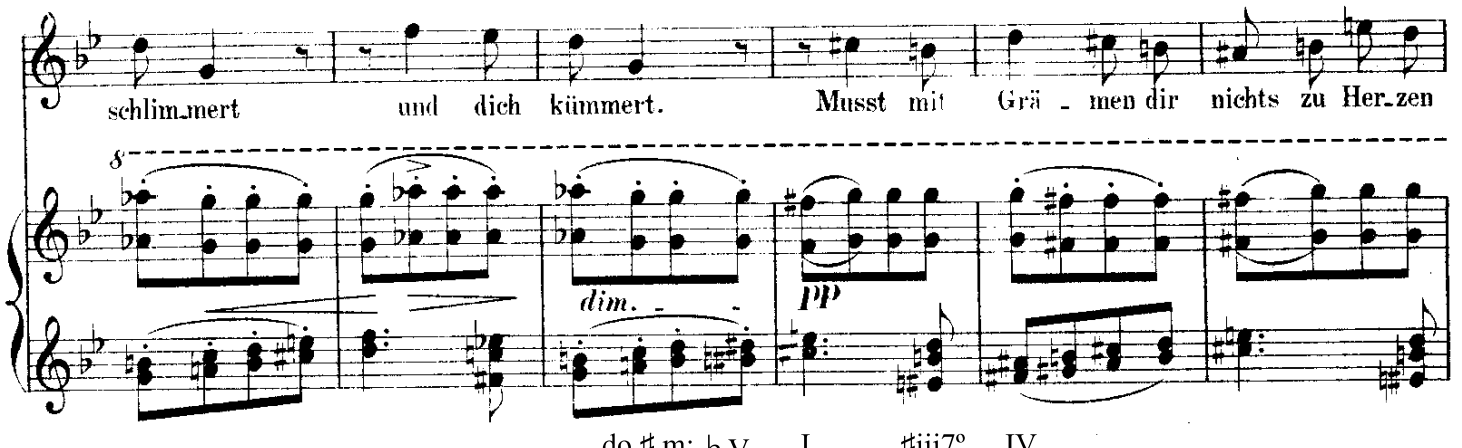
do \# m: bV I $\quad \# i i i 7^{\circ}$ IV
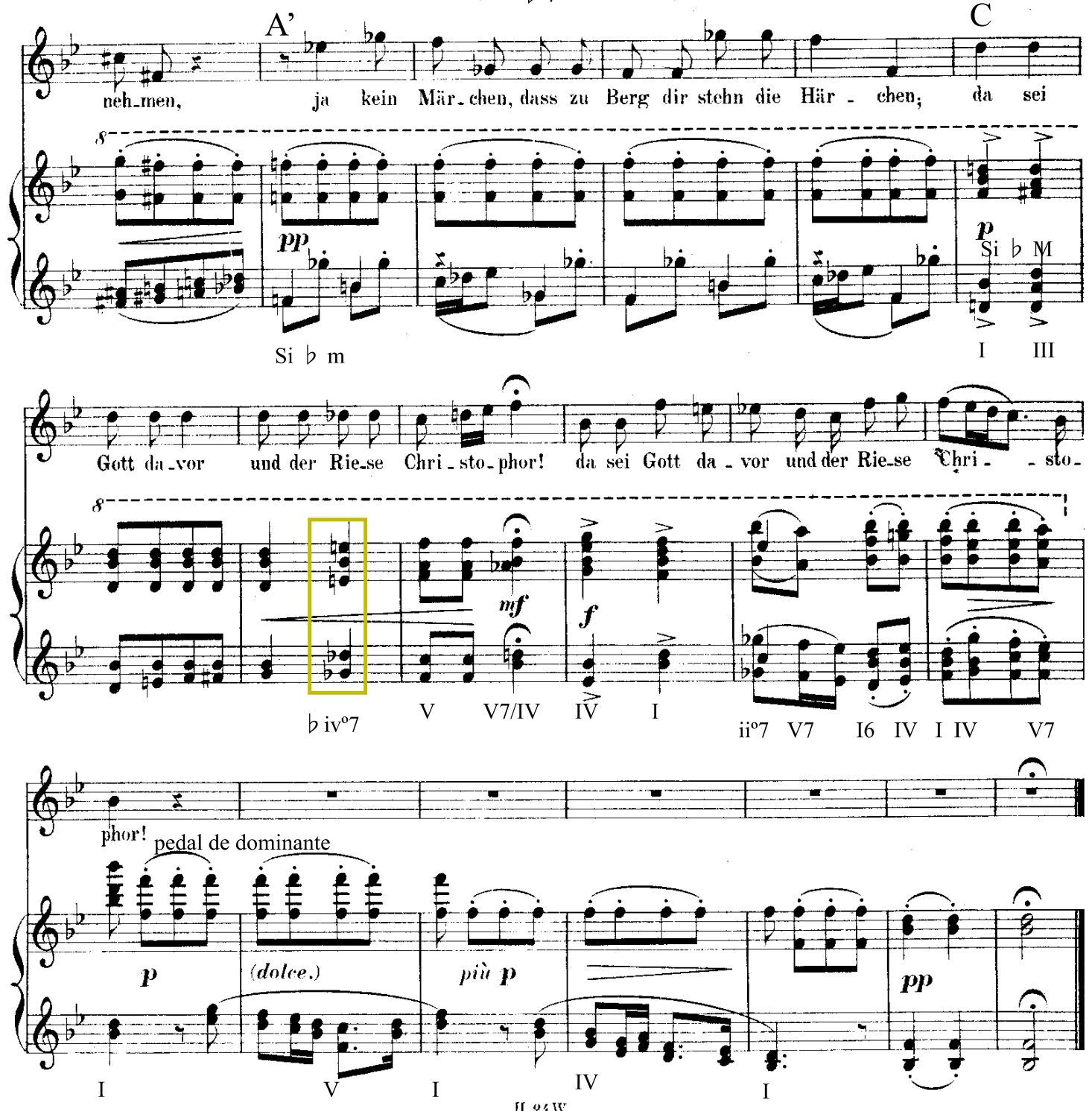
La DOBLE TRANSFormación DE UN TEXTo DE Miguel de CERVANTES EN UN POEMA ALEMÁN Y EN UN LIED: El ENSALMO DE PRECIOSA “CABECITA, CABECITA" DE LA NOVELA EJEMPlaR La GITANILLA

\section{BIBLIOGRAFÍA}

Böhl de Farber, J. N. (1821). Floresta de Rimas Antiguas Castellanas. Hamburgo: Perthes y Besser.

Cervantes Saavedra, M. (1613). "La gitanilla”, en Cervantes Saavedra, Miguel de. Novelas Ejemplares. Madrid: Juan de la Cuesta.

Cervantes Saavedra, M. (1799). "La gitanilla”, en Cervantes Saavedra, Miguel de. Novelas Ejemplares, Tomo I. Madrid: Imprenta de Villalpando.

Cervantes Saavedra, M. (2003). Obras completas I. Madrid: Santillana.

Domínguez Caparrós, J. (2002). Métrica de Cervantes. Alcalá de Henares: Centro de Estudios Cervantinos.

Fernández San Emeterio, G. (1997). "La pervivencia de la poesía española medieval y de los Siglos de Oro en la poesía alemana del siglo XIX". Dicenda. Cuadernos de Filología Hispánica, XV, pp. 203-217. Recuperado de:

http://revistas.ucm.es/index.php/DICE/article/view/DICE9797110203A/12848

[consultado el 17 de abril de 2017].

Fernández San Emeterio, G. (2005). "Canciones líricas españolas del Siglo de Oro y versiones musicales de Robert Schumann: El Spanisches Liederspiel y las Spanische Liebeslieder". eHumanista: Journal of Iberian Studies, V, pp. 187-194. Recuperado de:

http://www.ehumanista.ucsb.edu/volumes/volume 05/articles/Fernandez.pdf [consultado el 17 de abril de 2017].

García Albero, J. (2015). "Panorama de la traducción y recepción de las novelas ejemplares de Cervantes en la Alemania del siglo XIX”, en E. Marigno, C. Mata y H. Hernán (eds.). Cervantes creador y Cervantes recreado. Pamplona: Servicio de Publicaciones de la Universidad de Navarra, pp. 87-99. Recuperado de:

http://dadun.unav.edu/bitstream/10171/37692/1/CervantesCreador 06 GarciaAlbero.p df [consultado el 17 de abril de 2017].

Geibel, E. y Heise, P. (1852). Spanisches Liederbuch, Berlín: Hertz. 


\section{FrANCISCo PÉREZ SÁNCHEZ}

Geibel, E. y Heise, P. (1922). Der Briefwechsel von E. Geibel und P. Heyse, Múnich: Erich Petzet.

Geibel, E. (1843). Volkslieder und Romanzen der Spanier im Versmasse des Originals verdeutscht. Berlín: Verlag von Alexander Dunder. Recuperado de:

http://reader.digitale-sammlungen.de/de/fs1/object/display/bsb10607464 00007.html [consultado el 17 de abril de 2017].

Jestremski, M. (2011). Hugo-Wolf-Werkverzeichnis, Kassel/Stuttgart: Bärenreiter.

Kramer, L. (1996). "Hugo Wolf: Subjectivity in the Fin-de-Siécle Lied”, en R. Hallmark (ed.). German Lieder in the Nineteenth Century. Nueva York: Schirmer Books.

Montero Reguera, J. (2013). “La Gitanilla: una Rei-vindicación de la Poesía”. Ínsula, 799800, pp. 34-36.

Newman, E. (1915). "Hugo Wolf and the Lyric". The Musical Times, 56 no. 873, pp. 649651.

Salomon, W. (1924). Hugo Wolf als Liedkomponist. Eine stilkritische Untersuchung (Tesis doctoral). Frankfurt am Main: Universität Frankfurt am Main.

Sams, E. (1983). The songs of Hugo Wolf. ( $2^{\mathrm{a}}$ ed.). Londres: Eulenburg Books.

Schilhawsky, P. (2004). Wege zur Liedinterpretation. Viena/Múnich: Doblinger.

Sleeman, M. G. y Davies, G. A. (1982). "Variations on Spanish Themes. The «Spanisches Liederbuch» of Emanuel Geibel and Paul Heyse and its reflection in the songs of Hugo Wolf', en M. G. Sleeman y G. A. Davies (eds.). Proceedings of the Leeds Philosophical and Literary Society (Literary and Historical Section). Leeds: W. S. Maney and Son Ltd., pp. 155-274.

Thürmer, H. (1970). Die Melodik in den Liedern von Hugo Wolf, Schriften zur Musik, Band 2. Giebing Über Prien Am Chiemsee: Musikverlag Emil Katzbichler.

Vega Cernuda, M. A. y Wegene, H. (eds.) (2002). España y Alemania: Percepciones Mutuas de Cinco Siglos de Historia. Madrid: Editorial Complutense.

VV. AA. (2010). Hugo Wolf: Biographisches. Netzwerk. Rezeption. Viena: Metroverlag.

Walker, F. (1968). Hugo Wolf: A Biography (2a ed.). Londres: Dent.

Werba, E. (1984). Hugo Wolf und seine Lieder. Viena: Österreicher Bundesverlag. 
La DOBLE TRANSFORMACIÓN DE UN TEXTO DE Miguel De CERVANTES EN UN POEMA ALEMÁN Y EN UN LIED: El ENSALMO DE PRECIOSA “CABECITA, CABECITA" DE LA NOVELA EJEMPLAR LA GITANILLA

Youens, S. (1992). Hugo Wolf: The Vocal Music. Princeton: Princeton University Press.

Wolf, H. (1967/1994). Spanisches Liederbuch. Sämtliche Werke. Band 4. Kritische Gesamtausgabe. Viena: Musikwissenschaftlicher Verlag.

Fecha de recepción: 14/10/2017

Fecha de aceptación: 04/12/2017 